\title{
Correlation between histological grade and positron emission tomography parameters in cervical carcinoma
}

\author{
VANESSA MOCCIARO $^{1}$, PAOLO SCOLLO ${ }^{2}$, ALESSANDRO STEFANO ${ }^{1}$, STEFANIA GIERI $^{1}$, GIORGIO RUSSO ${ }^{1}$, \\ GIUSEPPE SCIBILIA $^{2}$, SEBASTIANO COSENTINO ${ }^{3}$, GABRIELLA MURE $^{3}$, SARA BALDARI $^{3}$, \\ MARIA GABRIELLA SABINI ${ }^{4}$, FILIPPO FRAGGETTA ${ }^{5}$, MARIA CARLA GILARDI $^{1}$ and MASSIMO IPPOLITO ${ }^{3}$ \\ ${ }^{1}$ Institute of Molecular Bioimaging and Physiology, National Research Council, Cefalù, I-90015 Palermo; Departments of \\ ${ }^{2}$ Gynecology and ${ }^{3}$ Nuclear Medicine; ${ }^{4}$ Medical Physics and ${ }^{5}$ Pathology Units, Cannizzaro Hospital, I-95126 Catania, Italy
}

Received June 5, 2015; Accepted May 12, 2016

DOI: $10.3892 / 01.2016 .4771$

\begin{abstract}
The aim of the present study was to evaluate the changes in cervical cancer glucose metabolism for different levels of cellular differentiation. The metabolic activity was measured by standardized uptake value (SUV), SUV normalized to lean body mass, metabolic tumor volume and total lesion glycolysis using fluorine-18 fluorodeoxyglucose positron emission tomography (PET). A correlation study of these values could be used to facilitate therapeutic choice and to improve clinical practice and outcome. This study considered 32 patients with diagnosed cervical cancers, at different International Federation of Gynecology and Obstetrics stages. Glucose metabolism was assessed by PET examination, and histological specimens were examined to determine their initial grade of differentiation. A correlation study of these values was evaluated. Histological examination showed that all cases were of squamous cell carcinoma. Regarding the differentiation of the tumor, 19 well- to moderately-differentiated tumors and 13 poorly-differentiated tumors were determined. Negative findings for correlations between metabolic parameters and initial grade of histological differentiation were found, and considering that histological grade has been shown to have no consistent prognostic value in cervical cancer treatment, PET imaging could play a significant role in cervical cancer prognosis.
\end{abstract}

\section{Introduction}

Cervical cancer is one of the most frequently occurring cancers in women and a significant cause of mortality with

Correspondence to: Dr Alessandro Stefano, Institute of Molecular Bioimaging and Physiology, National Research Council, Contrada Pietrapollastra, Cefalù, I-90015 Palermo, Italy

E-mail: alessandro.stefano@ibfm.cnr.it

Key words: cervical cancer, differentiation levels, glucose metabolism, positron emission tomography imaging, metabolic tumor volume
528,000 novel cancer cases and 266,000 cancer mortalities among females, worldwide in 2012. Despite advances in screening, cervical cancer remains a major health problem worldwide, with a high number of cases already at a locally advanced disease stage at presentation $(1,2)$. The histological subtype that most commonly occurs in $\sim 90 \%$ of cases is squamous cell carcinoma. Adenocarcinoma and adenosquamous carcinoma with unusual histologies, such as sarcoma and lymphoma, are quite rare ( $10 \%$ of cases) (3). Staging is mainly determined on the basis of tumor size in the cervix or in its extension into the pelvis. The current staging system is based on physical examination, but imaging used in addition to this provides more information regarding lesion size, adjacent organ invasion and distant metastases (4).

The International Federation of Gynecology and Obstetrics (FIGO) $(5,6)$ defines clinical stages of cervical cancer based on the tumor size and extension, the involvement of regional lymph nodes and the presence of distant metastases (7). In addition, cervical cancer is classified into three grades based on histological criteria. The grading is a measure of cell differentiation in the tumor, based on the resemblance of the tumor to the tissue of origin. The grade describes the appearance of the cancerous cells as follows: Grade 1 (G1; well-differentiated) and grade 2 (G2; moderately-differentiated) are the most common categories, whereas grade 3 (G3; poorly-differentiated) indicates an aggressive tumor. In addition, certain references in the literature support the fact that grading does not play a significant role in the prognosis $(8,9)$.

Positron emission tomography (PET) with fluorine-18 fluorodeoxyglucose (FDG) is an important tool for the diagnosis and staging of cervical cancer, providing metabolic information on the tumor (10). FDG-PET images have a fundamental impact in numerous fields of oncology, as metabolic changes are often faster and more indicative of the effects of therapy with respect to morphological changes, providing a more rapid evaluation of the response to treatment (11-13). PET is used together with computed tomography (CT), as the low spatial resolution of PET limits its capability in the evaluation of the primary tumor and in the precise anatomical localization of regions of increased focal uptake. The introduction of integrated PET/CT scanners has overcome these limitations $(14,15)$. In the clinical routine, PET lesions are analyzed using the standardized 
uptake value (SUV), a semi-quantitative parameter for discrimination between patients that respond or do not respond to treatment $(16,17)$. Since 1999, the European Organization for Research and Treatment of Cancer (EORTC) has proposed metabolic criteria to evaluate the treatment response by PET imaging based on SUV (18). Therapy response can be assessed using CT images according to Response Evaluation Criteria in Solid Tumors (19), depending on tumor anatomical changes, but these criteria do not reflect metabolic changes. The tumors detected by morphological imaging can include non-viable necrotic tissues. Thus, PET measurements can provide more accurate information than either $\mathrm{CT}$, magnetic resonance imaging (MRI) or 3-dimensional ultrasonography (3D US). In a recent review (20), the role of PET parameters in a number of cancer types, including cervical cancer, was studied. In particular, a low degree of FDG uptake may be associated with a good prognosis, as also confirmed by Kwee et al (21). The association between the FDG uptake degree and histology type was studied by Kidd et al (22), and it was concluded that the maximum SUV differed among the various subtypes of cervical cancer. Additional measures of tumor metabolic activity determined by PET images were proposed by Larson et al (23), including the volumetric measurement of tumor cells with increased FDG uptake. Metabolic volumes may be used both for treatment response monitoring and for target volume delineation in radiotherapy. Due to the nature of PET images (i.e., a low spatial resolution, high noise levels and weak boundaries), metabolic volume varies substantially depending on the algorithm used to delineate this. For this reason, various approaches for the metabolic volume calculation have been suggested (24). Visual delineation is the most commonly used technique in the clinical environment, as it is easily applicable, but it is also potentially inaccurate, as it is susceptible to the window level settings and subject to intra and inter-operator variability. For visual delineation, the physician must correctly identify the lesion and have excellent contrast between tumor and normal tissues. For this reason, an operator and scanner independent segmentation method is mandatory to accurately define the metabolic volume (24). Kim et al (25) evaluated 45 patients with maximum SUV and metabolic tumor volume (MTV), and MTV proved to be an important independent prognostic marker for disease-free survival. In other published data (26), it was suggested that maximum SUV and MTV could predict overall survival, recurrence-free survival and disease-specific survival. Nevertheless, the measurement of PET volumes provides only volumetric information and does not assess metabolic activity; parameters that combine the volumetric and metabolic information, such as the total lesion glycolysis (TLG), can provide a better evaluation of prognosis. A number of studies have shown the usefulness of TLG as a predictive parameter to guide treatment decisions in different cancers (27-31). Maximum SUV, MTV and TLG may be considered as predictive and risk stratification markers for the management of cervical cancer patients (4). The prognostic value of metabolic and volumetric PET parameters in cervical cancer was investigated in a study by Yoo et al (32), which showed that TLG may be a significant independent prognostic factor for event-free survival and that it may be considered more effective than the other PET parameters in determining patient prognosis. In another study by Miccò et al (33),
MTV and TLG were associated with high-risk features and were indicated to serve as prognostic biomarkers of survival in patients with cervical cancer. Finally, previous studies assessed the usefulness of PET studies in cervical carcinoma staging and showed that high FDG uptake does not correlate with the initial grade of histological differentiation (34,35). As the grade has no consistent prognostic value in cervical cancer treatment $(8,9)$, FDG uptake-based parameters could provide a better evaluation of the prognosis in cervical cancer.

The aim of the present study was to evaluate the correlation between the PET parameters and the different levels of differentiation in patients with cervical cancer. This study could be useful for detecting PET prognostic factors that affect patient management decisions, improving the clinical outcome.

\section{Patients and methods}

Patients. This study considered data from 32 patients, aged 24-69 years, who were diagnosed with cervical cancer at FIGO stages IB-IVB. The patients were recruited between July 2011 and July 2012 at the Department of Gynecology, Cannizzaro Hospital (Catania, Italy). Pre-treatment evaluation included taking the patient's history, a physical examination, a vaginal-pelvic examination, a cervical biopsy and complete blood analysis. Tumor extension was assessed clinically by abdominal MRI, 3D US and PET examinations. Histological specimens were also examined to determine the initial grade of differentiation. Diagnosis and grading were rendered according to World Health Organization criteria (36).

PET/CT imaging. PET/CT examinations were performed at Cannizzaro Hospital using the Discovery 690 system with time-of-flight (GE Healthcare, Little Chalfont, UK) and the Gemini GXL system (Philips Healthcare, Surrey, UK). The weight of each patient was measured on the day of the PET/CT scan. The actual injected and residual radioactive doses of the radiotracer were measured.

For Discovery 690 system examinations, patients fasted for $6 \mathrm{~h}$ prior to the PET/CT scans, and only patients with a serum glucose level of $<200 \mathrm{mg} / \mathrm{dl}$ were injected intravenously with FDG (37 MBq/10 kg). PET/CT acquisition started 50-60 min after injection. Patients were positioned with their arms raised during the PET/CT examinations. All studies were performed according to a standard protocol, scanning from the top of the skull to the middle of the thigh, which included a scout scan at $10 \mathrm{~mA}$, a CT scan at $120 \mathrm{kVp}$ and 50-120 $\mathrm{mA}$, and a 3D whole-body PET scan ( $2 \mathrm{~min} /$ bed position). PET images were reconstructed using the $3 \mathrm{D}$ ordered subset expectation maximization algorithm optimized to time-of-flight, with corrections for random, scatter and attenuation. For each bed position, the CT volume consisted of $512 \times 512$ voxels of $1.36 \times 1.36 \times 3.75 \mathrm{~mm}^{3}$ in size, while the PET image volume consisted of $256 \times 256$ voxels of $2.73 \times 2.73 \times 3.27 \mathrm{~mm}^{3}$ in size.

For Gemini system analysis, the patients fasted for $6 \mathrm{~h}$ prior to PET/CT scans being performed, and only patients with a serum glucose level of $<200 \mathrm{mg} / \mathrm{dl}$ were injected intravenously with FDG (3 MBq/kg of body weight, normalized to body mass index, with a minimum of $148 \mathrm{MBq}$ and a maximum of $296 \mathrm{MBq}$ ). The weight of each patient was measured on the same weighing machine on the day of the PET/CT scan. The 
actual injected and residual radioactive doses were measured by a dose measurement system. PET/CT acquisition started 50-60 min after radiotracer injection. Patients were positioned with their arms raised and were allowed to breathe normally during the PET/CT examinations. All studies were performed according to a standard protocol, scanning from the top of the skull to the middle of the thigh, which included a scout scan at $20 \mathrm{~mA}$, a CT scan at $120 \mathrm{kVp}$ and $60 \mathrm{~mA}$, and a 3D whole-body PET scan ( $2 \mathrm{~min} /$ bed position). PET images were reconstructed using the line of response row action maximum likelihood algorithm, with corrections for random, scatter and attenuation. For each bed position, the CT volume consisted of $512 \times 512$ voxels of $1.17 \times 1.17 \times 5 \mathrm{~mm}^{3}$ in size, while the PET image volume consisted of $144 \times 144$ voxels of $4 \times 4 \times 4 \mathrm{~mm}^{3}$ in size.

PET/CT data analysis. Maximum SUV normalized to body weight (SUVmax), SUV normalized to lean body mass (SUL), MTV and TLG were determined from FDG-PET images in the primary cervical tumor of each patient.

Volumetric and semi-quantitative parameters were extracted using a software package that has been developed and implemented 'ad hoc' by our group to assess treatment response in oncological patients: PET parameters were measured on the lesions that had previously been identified as suspicious by visual analysis using our automatic segmentation algorithm based on random walks on graphs (37). The nuclear medicine physician interactively selected the PET region with uptake higher than the background, excluding any physiological uptake findings, by clicking on the image. Lesion contour was automatically plotted, and MTV was calculated. SUVmax was obtained from the maximum pixel value in the MTV (18). SUL was obtained as the SUV normalized to lean body mass calculated as the average within a $1-\mathrm{cm}^{3}$ spherical volume of interest, centered on the portion of the lesion with the highest uptake, according to the PET Response Criteria In Solid Tumors (PERCIST) (11). Finally, TLG, which combines the volumetric and metabolic information of PET lesions, was calculated as the product of the MTV with its mean SUV (23).

Statistical data analysis. To compare tumor aggressiveness resulting from PET quantitative values and histological examinations, Student's t-test was used. Student's t-test was therefore performed to compare SUVmax, SUL and MTV for each of the degrees of differentiation. To find eventual correlations between uptake values and histological grade, Spearman's correlation coefficient was used. Regression analysis was applied for comparing PET volumetric measurements (MTV and TLG) with semi-quantitative measurements (SUVmax and SUL). $\mathrm{P}<0.05$ was considered to indicate a statistically significant difference. Statistical evaluations were performed using MedCalc software, version 12.3.0.0 (Frank Schoonjans, Mariakerke, Belgium).

\section{Results}

Patients and lesion characteristics. Tumors were graded into G1, G2, G3 according to well defined criteria (38). Examinations determined 19 well to moderately-differentiated tumors (1 G1 and $18 \mathrm{G} 2$ ) and 13 poorly-differentiated tumors (G3).
Table I. Patient characteristics.

\begin{tabular}{lc}
\hline Characteristic & Value \\
\hline Age, years & \\
Mean & 50.31 \\
Range & $24-69$ \\
Histology, n (\%) & \\
Squamous cell carcinoma & $32(100.00)$ \\
FIGO stage, n (\%) & \\
I & $1(3.13)$ \\
II & $28(87.50)$ \\
III & $1(3.13)$ \\
IV & $2(6.25)$ \\
Tumor grade, n (\%) & \\
Well- to moderately-differentiated & $19(59.38)$ \\
Poorly-differentiated & $13(40.63)$
\end{tabular}

FIGO, International Federation of Gynecology and Obstetrics.

All patients presented with squamous cell carcinoma and the majority of patients $(87.5 \%)$ had FIGO stage IIB disease (Table I).

Analysis of PET images (8 examinations performed using the Discovery 690 system and 24 using the Gemini GXL) showed an SUVmax of 15 \pm 3.92 (range, 8.14-23.93; median, 14.72), an SUL of 10.30 \pm 2.37 (range, 6.28-16.74; median, 10.35), an MTV of $43.92 \pm 327.90 \mathrm{ml}$ (range, 7.87-109.49 ml; median, $33.8 \mathrm{ml}$ ) and a TLG of $328.5 \pm 2208.99 \mathrm{ml}$ (range, 45.75-782.77 ml; median, $269.06 \mathrm{ml}$ ). Representative PET images for three patient studies are shown in Fig. 1.

Correlation analysis. Fig. 2 shows the scatter plots for PET values and grade of histological differentiation. Student's t-test for unpaired data was used for comparing the mean SUVmax for the two levels of tumor differentiation (well- to moderately-differentiated vs. poorly-differentiated). The $\mathrm{t}$-test gave a $\mathrm{P}$-value of $>0.05(\mathrm{P}=0.9536)$, thus there was no significant difference in the SUVmax of well- to moderately-differentiated and poorly-differentiated cervical tumors. Student's t-test showed no significant difference for SUL vs. grading $(\mathrm{P}=0.7952)$, for MTV vs. grading $(\mathrm{P}=0.9049)$ or for TLG vs. grading $(\mathrm{P}=0.8335)$.

The mean SUVmax was $15.43 \pm 4.56$ for well- to moderately-differentiated cervical tumors and $15.34 \pm 4.42$ for poorly-differentiated tumors. The mean SUL was $9.53 \pm 2.84$ for G1 and G2, and 9.28 \pm 2.36 for G3. The mean MTV was $44.42 \pm 28.71 \mathrm{ml}$ for well- to moderately-differentiated cervical tumors and $43.19 \pm 27.81 \mathrm{ml}$ for poorly-differentiated tumors. The mean TLG was $335.08 \pm 215.91 \mathrm{ml}$ for $\mathrm{G} 1$ and $\mathrm{G} 2$, and $318.88 \pm 206.73 \mathrm{ml}$ for $\mathrm{G} 3$.

No positive association was found with Spearman's correlation coefficients or rank correlation between SUVmax and grade of histological differentiation $(\mathrm{P}=0.8957$; rho $=0.024)$, between SUL and histological grade $(\mathrm{P}=0.9701$; rho=0.007), or between MTV and histological grade $(\mathrm{P}=0.8957$, rho=$0.024)$, or between TLG and histological grade $(\mathrm{P}=0.8957$, 


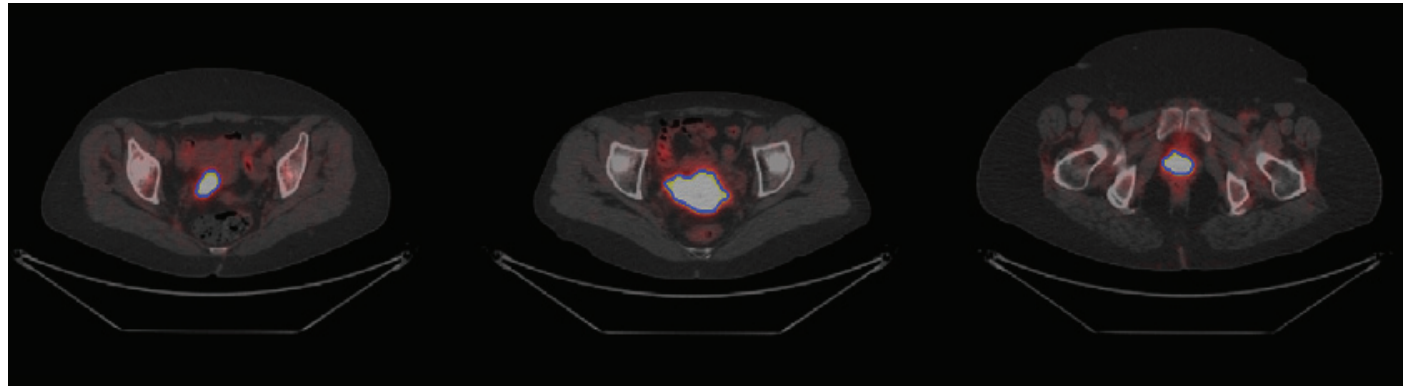

Figure 1. Three representative positron emission tomography/computed tomography image slices. FIGO stage, grade of histological differentiation, SUVmax, SUL, MTV and TLG were determined for each patient: (A) FIGO IIB, G3, SUVmax=14.20, SUL=8.96, MTV=17.81cc, TLG=108.40. (B) FIGO IIIB, G2, SUVmax=15.40, SUL=10.34, MTV=57.36cc, TLG=487.62. (C) FIGO IIB, G3, SUVmax=17.82, SUL=9.53, MTV=27.4cc, TLG=245.68. Segmentation results (blue contour) are also shown. FIGO, International Federation of Gynecology and Obstetrics; SUVmax, maximum standardized uptake value; SUL, SUV normalized to lean body mass; MTV, metabolic tumor volume; TLG, total lesion glycolysis.

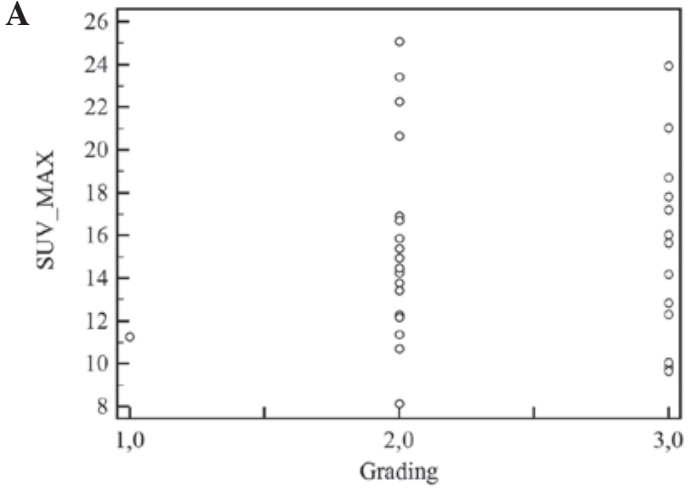

B
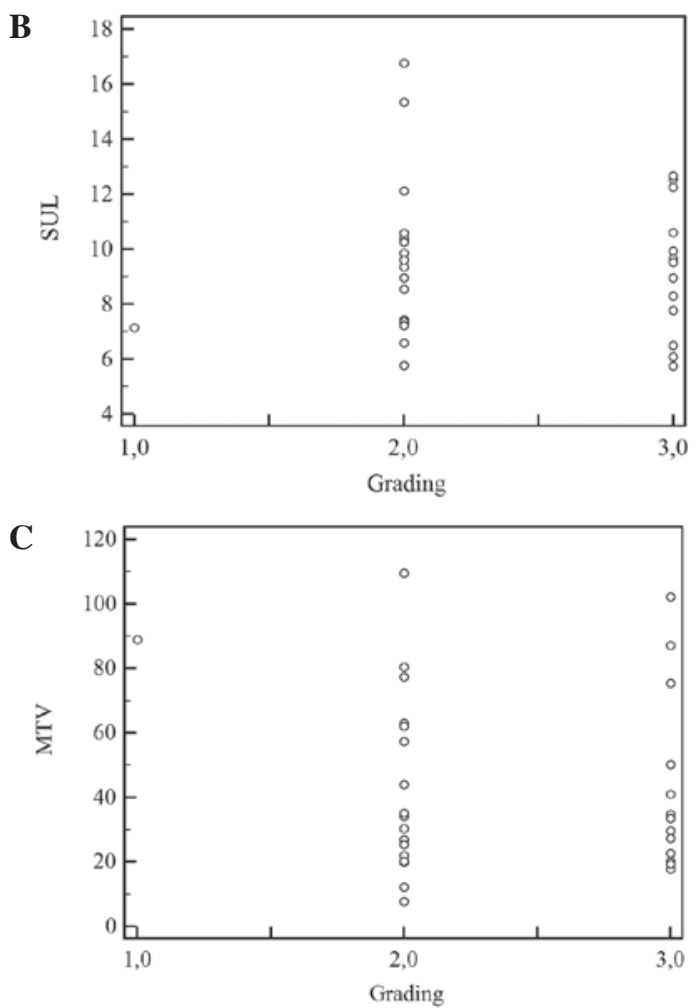

Figure 2. (A) Scatter plot comparing SUVmax and grade of histological differentiation (mean difference, $-0.095 ; 95 \%$ CI, -3.4066-3.2161; $\mathrm{t}=-0.0587$; $\mathrm{df}=30 ; \mathrm{P}=0.9536$ ). (B) Scatter plot comparing SUL and grade of histological differentiation (mean difference, $-0.2507 ; 95 \%$ CI, -2.2057-1.7043; $\mathrm{t}=-0.262$; $\mathrm{df}=30 ; \mathrm{P}=0.7952$ ). (C) Scatter plot comparing MTV and grade of histological differentiation (mean difference, -1.23 ; 95\% CI, -22.0711-19.6111; $\mathrm{t}=-0.121$; $\mathrm{df}=30 ; \mathrm{P}=0.9049$ ). SUVmax, maximum standardized uptake value; SUL, SUV normalized to lean body mass; MTV, metabolic tumor volume; CI, confidence interval; df, degrees of freedom.

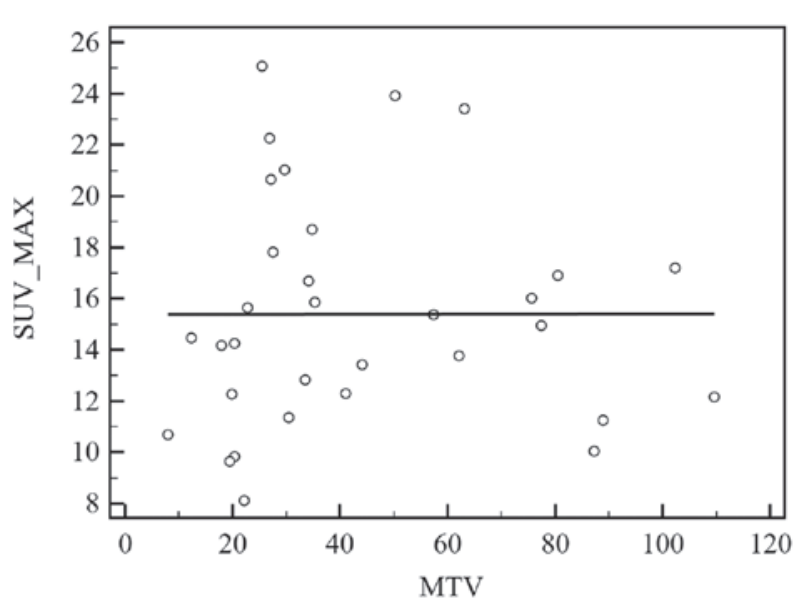

Figure 3. Regression plot comparing the association between SUVmax and PET tumor volume. SUVmax, maximum standardized uptake value; MTV, metabolic tumor volume.

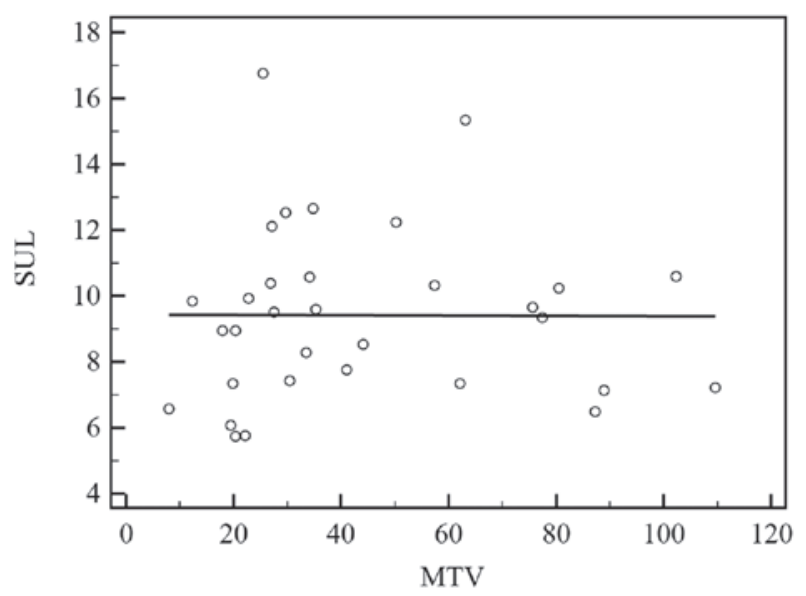

Figure 4. Regression plot comparing the association between SUL and PET tumor volume. SUL, standardized uptake value normalized to lean body mass; MTV, metabolic tumor volume.

rho=-0.024).It was concluded that there is no significant correlation between uptake values and the grade of histological differentiation.

Regression analysis was used to describe the association between SUVmax and MTV $\left(\mathrm{R}^{2}<0.00001\right)$ (Fig. 3), and 


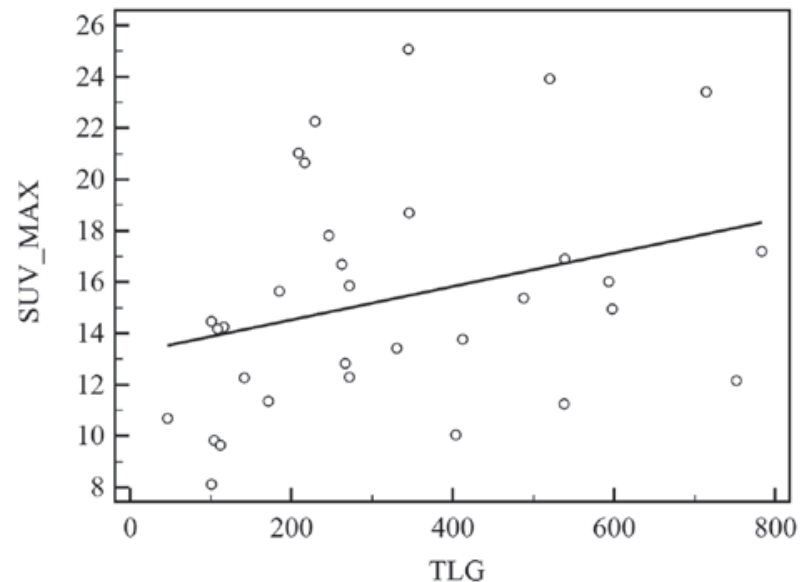

Figure 5. Regression plot comparing the association between cervical SUV and TLG. SUVmax, maximum standardized uptake value; TLG, total lesion glycolysis.

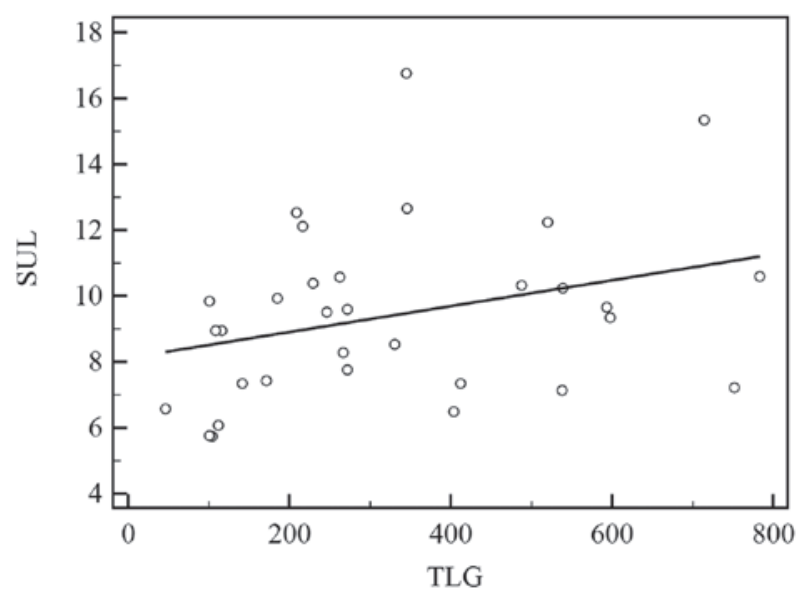

Figure 6. Regression plot comparing the association between cervical SUL and TLG. SUL, standardized uptake value normalized to lean body mass; TLG, total lesion glycolysis.

between SUL and MTV $\left(\mathrm{R}^{2}<0.00001\right)$ (Fig. 4). In each case, there was no correlation. The association between TLG and SUVmax (Fig. 5), and between TLG and SUL (Fig. 6) also showed no correlation. In addition, no correlation was found between grade and PET parameters when considering the following subgroups separately: i) 28 patients with the prevailing FIGO stage (IIB) $(\mathrm{P}>0.60)$; ii) 24 patients undergoing PET examination using the Gemini GXL ( $\mathrm{P}>0.14)$; and iii) 8 patients undergoing PET examination using the Discovery 690 system $(\mathrm{P}>0.58)$; only the SUL and grading comparison showed a $\mathrm{P}$-value of $<0.05(\mathrm{P}=0.039)$. This correlation should be further investigated in a larger sample.

\section{Discussion}

Medical imaging plays an important role in the assessment of patients with cervical cancer, from the diagnosis to the evaluation of the disease extent. The traditional prognostic factors, including lymph node metastasis, parametrial invasion, lesion size and tumor differentiation, provide significant clinical and pathological information. Therefore, certain studies have shown that PET imaging is useful for the assessment of prognosis in cervical cancer patients, by identifying the primary tumor and potential distant metastases, and allowing the risk stratification of patients $(4,10,32,33)$. Research efforts have focused on the identification of novel PET prognostic factors: An accurate prediction of prognosis is important, as it affects patient management decisions. In the clinical routine, PET images are analyzed semi-quantitatively using the SUV, a potential predictive biomarker for discrimination between patients who respond to treatment and those who do not. A number of studies have suggested that SUV is associated with prognosis in patients with cervical cancer $(4,33,39-41)$. In a study by Xue et al (42), the avidity of FDG uptake in primary cervical tumors was a predictor of survival outcome. Tumors that had a high SUVmax had a worse survival outcome than those with a low SUVmax. These findings suggest that patients with a high SUV may be at increased risk for disease recurrence and may benefit from more aggressive therapy. SUVmax is often considered the best measure of tumor uptake when considering that it is the most resistant to partial volume effect and is operator-independent (43), but it is highly variable due to the high noise level in PET data. In addition, SUVmax corresponds to the maximum pixel value in the lesion, and a single pixel may not be representative of the overall lesion uptake (16). For this reason, the study of alternative PET parameters could be useful. PERCIST introduces the SUL obtained from a fixed region of interest, obtaining an average of multiple pixel data, to remove interobserver variation and noise susceptibility. In addition, the use of volumetric parameters, as MTV and TLG, should be mandatory. The identification of effective PET prognostic factors can be obtained using a standardized PET imaging protocol consistent with the National Cancer Institute recommendations (44) and those of the Netherlands multicenter trial group, on well-calibrated and well-maintained PET scanners (45). In addition, in the evaluation of patients with gynecological malignancies, the role of PET parameters correlated with histological results may become central for an accurate diagnosis in order to achieve better treatment selection and planning.

The purpose of the present study was to investigate the association between initial histological grade and potential PET prognostic factors in 32 patients with cervical cancer at FIGO stages IB-IVB. The evaluation included physical and vaginal-pelvic examinations, a cervical biopsy, and MRI, 3D US and PET examinations prior to treatment.

Through the statistical tests, the present study found that PET values in the primary cervical tumor were not correlated with the initial grade of histological differentiation, independent of the PET system and the FIGO staging. Only the comparison between SUL and grading differentiation showed a $\mathrm{P}$-value of $<0.05$ in 8 patients undergoing PET Discovery 690 system examination. Nevertheless, this correlation should be verified on a larger study considering the small sample size. The usefulness of FDG-PET in the staging of squamous cervical carcinoma has already been studied and shows a high accumulation of FDG, but no correlation with the initial grade of histological differentiation $(34,35)$, as confirmed by the present study: Metabolic and volumetric measurements of FDG uptake did not result in 
a direct assessment of cervical cancer grade. As the grade has no consistent prognostic value in cervical cancer treatments, as reported in the literature $(8,9)$, PET parameters could provide a better evaluation of the prognosis. However, the present correlation results differ from those of previously published data. In a recent study (46), the quantitative analysis of PET/MRI results revealed a significant correlation between SUV and differentiation grade, which was in accordance with the results of the study by Kurokawa et al (47). In one study (25), the MTV was compared with pathological and clinical prognostic factors in 45 patients with stage IA-IIB cervical cancer. Statistical analysis showed that MTV was a better predictor of disease-free survival than traditional prognostic factors. It was suggested that patients with a high MTV may benefit from more aggressive treatment. In addition, MTV significantly differed among the groups according to tumor differentiation. MTV represents the metabolic extent of the oncological lesion and the size of the viable tumor cells, while TLG combines volumetric and SUV information, providing a better evaluation of the prognosis. TLG therefore appears to be of greater importance for patient prognosis in patients with cervical cancer (32).

The presence of contrasting results indicates that this is a complex field of research that requires prospective studies with a larger number of patients to evaluate the prognostic usefulness of metabolic and volumetric PET parameters. The main limitation of the present study is the low number of patients used to describe the possible correlation between histological grade and PET parameters in cervical carcinoma. This limitation is due to the requirement for a multidisciplinary team. Taking into consideration the importance that this study can have with regard to oncological patient management, additional analyses, such as randomized trials and larger studies, are required. In addition, further investigations should be performed monitoring the treatment response of these patients (48), defined as complete, partial, stable or progressive according to metabolic criteria such as EORTC and PERCIST, as well as MTV and TLG percentage changes.

Another limitation of the present study is the arbitrary choice of the segmentation method used to delineate the MTV. The MTV and consequently, the TLG, vary substantially depending on the algorithm used to delineate metabolic lesions, due to the low contrast and high noise level of PET images. An accurate and robust segmentation method is mandatory, as manual delineation is a prohibitively laborious task and considerable variations may occur among nuclear medicine physicians. Several semi-automatic methods have been proposed in the literature for MTV delineation and the choice of a standard method is a challenging yet unresolved step. For this reason, our automatic segmentation algorithm based on random walks on graphs was used (37).

The aim of the present study was to investigate the potential correlation between PET parameters and the initial grade of histological differentiation in cervical cancer. Negative findings were found independently of the PET system and the FIGO staging, and since the histological grade has no consistent prognostic value in cervical cancer treatment, PET imaging could play a significant role in cervical cancer prognosis, impacting patient management decisions.

\section{Acknowledgements}

This study was partially supported by the Italian National Operational Programme for Research and Competitiveness 2007-2013 (project ID, PON02_00643_3604826).

\section{References}

1. Parkin DM, Pisani P and Ferlay J: Global cancer statistics. CA Cancer J Clin 49: 33-64, 1999.

2. Rose PG: Chemoradiotherapy: The new standard care for invasive cervical cancer. Drugs.60: 1239-1244, 2000.

3. Iyer RB, Balachandran A and Devine CE: PET/CT and cross sectional imaging of gynecologic malignancy. Cancer Imaging 7: S130-S138, 2007.

4. Mirpour S, Mhlanga JC, Logeswaran P, Russo G, Mercier G and Subramaniam RM: The role of PET/CT in the management of cervical cancer. AJR Am J Roentgenology 201: W192-W205, 2013.

5. Quinn MA, Benedet JL, Odicino F, Maisonneuve P, Beller U, Creasman WT, Heintz AP, Ngan HY and Pecorelli S: Carcinoma of the cervix uteri. FIGO 26th annual report on the results of treatment in gynecological cancer. Int J Gynaecol Obstet 95 (Suppl 1): S43-S103, 2006.

6. Guo L, Liu X, Wang L, Sun H, Huang K, Li X, Tang F, Li S, Yuan X and Wang C: Outcome of international federation of gynecology and obstetrics stage iib cervical cancer from 2003 to 2012: An evaluation of treatments and prognosis: A retrospective study. Int J Gynecol Cancer 25: 910-918, 2015.

7. Ellenson LH and Wu TC: Focus on endometrial and cervical cancer. Cancer Cell 5: 533-538, 2004.

8. Zaino RJ, Ward S, Delgado G, Bundy B, Gore H, Fetter G, Ganjei P and Frauenhoffer E: Histopathologic predictors of the behavior of surgically treated stage IB squamous cell carcinoma of the cervix. A Gynecologic Oncology Group study. Cancer 69: 1750-1758, 1992

9. Baalbergen A, Ewing-Graham PC, Hop WC, Struijk P and Helmerhorst TJ: Prognostic factors in adenocarcinoma of the uterine cervix. Gynecologic Oncol 92: 262-267, 2004.

10. Zhao Q, Feng Y, Mao X and Qie M: Prognostic value of fluorine-18-fluorodeoxyglucose positron emission tomography or PET-computed tomography in cervical cancer: A meta-analysis. Int J Gynecol Cancer 23: 1184-1190, 2013.

11. Wahl RL, Jacene H, Kasamon Y and Lodge MA: From RECIST to PERCIST: Evolving considerations for PET response criteria in solid tumors. J Nucl Med 50 (Suppl 1): S122-S150, 2009.

12. Michaelis LC and Ratain MJ: Measuring response in a post-RECIST world: From black and white to shades of grey. Nat Rev Cancer 6: 409-414, 2006.

13. Stefano A, Russo G, Ippolito M, Cosentino S, Murè G, Baldari S, Sabini MG, Sardina D, Valastro LM, Bordonaro R, et al: Evaluation of erlotinib treatment response in non-small cell lung cancer using metabolic and anatomic criteria. Q J Nucl Med Mol Imaging: May 9, 2014 (Epub ahead of print).

14. Heron DE, Andrade RS, Beriwal S and Smith RP: PET-CT in radiation oncology: The impact on diagnosis, treatment planning and assessment of treatment response. Am J Clin Oncol 31: 352-362, 2008

15. Bar-Shalom R, Yefremov N, Guralnik L, Gaitini D, Frenkel A, Kuten A, Altman H, Keidar Z and Israel O: Clinical performance of PET/CT in evaluation of cancer: Additional value for diagnostic imaging and patient management. J Nuc Med 44: 1200-1209, 2003.

16. Tanvetyanon T,Eikman EA, Sommers E, Robinson L, Boulware D and Bepler G: Computed tomography response, but not positron emission tomography scan response, predicts survival after neoadjuvant chemotherapy for resectable non-small-cell lung cancer. J Clin Oncol 26: 4610-4616, 2008.

17. Hoekstra CJ, Stroobants SG, Smit EF, Vansteenkiste J, van Tinteren H, Postmus PE, Golding RP, Biesma B, Schramel FJ, van Zandwijk N, et al: Prognostic relevance of response evaluation using (18F)-2-fluoro-2-deoxy-D-glucose positron emission tomography in patients with locally advanced non-small-cell lung cancer. J Clin Oncol 23: 8362-8370, 2005.

18. Young H, Baum R, Cremerius U, Herholz K, Hoekstra O, Lammertsma AA, Pruim J and Price P: Measurement of clinical and subclinical tumour response using (18F)-fluorodeoxyglucose and positron emission tomography: Review and 1999 EORTC recommendations. European organization for research and treatment of cancer (EORTC) PET study group. Eur J Cancer 35: 1773-1782, 1999. 
19. Eisenhauer EA, Therasse P, Bogaerts J, Schwartz LH, Sargent D, Ford R, Dancey J, Arbuck S, Gwyther S, Mooney M, et al: New response evaluation criteria in solid tumours: Revised RECIST guideline (version 1.1). Eur J Cancer 45: 228-247, 2009.

20. Rahim MK, Kim SE, So H, Kim HJ, Cheon GJ, Lee ES, Kang KW and Lee DS: Recent Trends in pet image interpretations using volumetric and texture-based quantification methods in nuclear oncology. Nucl Med Mol Imaging 48: 1-15, 2014.

21. Kwee TC, Basu S, Saboury B, Ambrosini V, Torigian DA and Alavi A: A new dimension of FDG-PET interpretation: Assessment of tumor biology. Eur J Nucl Med Mol Imaging 38: 1158-1170, 2011

22. Kidd EA, Spencer CR, Huettner PC, Siegel BA, Dehdashti F, Rader JS and Grigsby PW: Cervical cancer histology and tumor differentiation affect $18 \mathrm{~F}$-fluorodeoxyglucose uptake. Cancer 115: 3548-3554, 2009.

23. Larson SM, Erdi Y, Akhurst T, Mazumdar M, Macapinlac HA, Finn RD, Casilla C, Fazzari M, Srivastava N, Yeung HW, et al: Tumor treatment response based on visual and quantitative changes in global tumor glycolysis using PET-FDG imaging. The visual response score and the change in total lesion glycolysis. Clin Positron Imaging 2: 159-171, 1999.

24. Zaidi $\mathrm{H}$ and El Naqa I: PET-guided delineation of radiation therapy treatment volumes: A survey of image segmentation techniques. Eur J Nucl Med Mol Imaging 37: 2165-2187, 2010.

25. Kim BS, Kim IJ, Kim SJ, Nam HY, Pak KJ, Kim K and Yun MS The prognostic value of the metabolic tumor volume in FIGO stage IA to IIB cervical cancer for tumor recurrence: Measured by F-18 FDG PET/CT. Nucl Med Mol Imaging 45: 36-42, 2011.

26. Kidd EA, El Naqa I, Siegel BA, Dehdashti F and Grigsby PW: FDG-PET-based prognostic nomograms for locally advanced cervical cancer. Gynecol Oncol 127: 136-140, 2012.

27. Lee HY, Hyun SH, Lee KS, Kim BT, Kim J, Shim YM, Ahn MJ, Kim TS, Yi CA and Chung MJ: Volume-based parameter of 18) F-FDG PET/CT in malignant pleural mesothelioma: Prediction of therapeutic response and prognostic implications. Ann Surg Oncol 17: 2787-2794, 2010.

28. Costelloe CM, Macapinlac HA, Madewell JE, Fitzgerald NE, Mawlawi OR, Rohren EM, Raymond AK, Lewis VO, Anderson PM, Bassett RL Jr, et al: 18F-FDG PET/CT as an indicator of progression-free and overall survival in osteosarcoma. J Nucl Med 50: 340-347, 2009.

29. Xie P, Yue JB, Zhao HX, Sun XD, Kong L, Fu Z and Yu JM: Prognostic value of 18F-FDG PET-CT metabolic index for nasopharyngeal carcinoma. J Cancer Res Clin Oncol 136: 883-889, 2010

30. Yoo J, Choi JY, Lee KT, Heo JS, Park SB, Moon SH, Choe YS, Lee KH and Kim BT: Prognostic Significance of volume-based metabolic parameters by (18)F-FDG PET/CT in Gallbladder Carcinoma. Nucl Med Mol Imaging 46: 201-206, 2012.

31. Stefano A: Metabolic response assessment in non-small cell lung cancer patients after platinum-based therapy: A preliminary analysis. Curr Med Imaging Rev 11: 218-217, 2015.

32. Yoo J, Choi JY, Moon SH, Bae DS, Park S, Choe YS, Lee KH and Kim BT: Prognostic significance of volume-based metabolic parameters in uterine cervical cancer determined using $18 \mathrm{~F}$-fluorodeoxyglucose positron emission tomography. Int J Gynecol Cancer 22: 1226-1233, 2012.

33. Miccò M, Vargas HA, Burger IA, Kollmeier MA, Goldman DA Park KJ, Abu-Rustum NR, Hricak $\mathrm{H}$ and Sala E: Combined pre-treatment MRI and 18F-FDG PET/CT parameters as prognostic biomarkers in patients with cervical cancer. Eur J Radiol 83: 1169-1176, 2014

34. Liao S, Lan X, Cao G, Yuan H and Zhang Y: Prognostic predictive value of total lesion glycolysis from $18 \mathrm{~F}-\mathrm{Fdg} \mathrm{Pet} / \mathrm{Ct}$ in post-surgical patients with epithelial ovarian cancer. Clin Nucl Med 38: 715-720, 2013.
35. Yen TC, See LC, Lai CH, Yah-Huei CW, Ng KK, Ma SY, Lin WJ, Chen JT, Chen WJ, Lai CR and Hsueh S: 18F-FDG uptake in squamous cell carcinoma of the cervix is correlated with glucose transporter 1 expression. J Nucl Med 45: 22-29, 2004.

36. Park JO, Lee Sl, Song SY, Kim K, Kim WS, Jung CW, Park YS, Im YH, Kang WK, Lee MH, et al: Measuring response in solid tumors: Comparison of RECIST and WHO response criteria. Jpn J Clin Oncol 33: 533-537, 2003.

37. Stefano A, Vitabile S, Russo G, Ippolito M, Sardina D, Sabini MG, et al: A graph-based method for PET image segmentation in radiotherapy planning: A pilot study. In: Image Analysis and Processing - ICIAP 2013. Petrosino A (ed). Springer-Verlag, Berlin, pp711-720, 2013.

38. Kurman JR: Tumors of the cervix, vagina and vulva. Afip Atlas of Tumor Pathology. Vol 5. 4th edition. Silverberg SG (ed). ARP Press Washington, WA, USA, pp 114, 2010.

39. Kidd EA, Siegel BA, Dehdashti F and Grigsby PW: The standardized uptake value for F-18 fluorodeoxyglucose is a sensitive predictive biomarker for cervical cancer treatment response and survival. Cancer 110: 1738-1744, 2007.

40. Husain A, Akhurst T, Larson S, Alektiar K, Barakat RR and Chi DS: A prospective study of the accuracy of 18Fluorodeoxyglucose positron emission tomography (18FDG PET) in identifying sites of metastasis prior to pelvic exenteration. Gynecol Oncol 106: 177-180, 2007.

41. Konishi H, Takehara K, Kojima A, Okame S, Yamamoto Y, Shiroyama Y, Yokoyama T, Nogawa T and Sugawara Y: Maximum standardized uptake value of fluorodeoxyglucose positron emission tomography/computed tomography is a prognostic factor in ovarian clear cell adenocarcinoma. Int J Gynecol Cancer 24: 1190-1194, 2014.

42. Xue F, Lin LL, Dehdashti F, Miller TR, Siegel BA and Grigsby PW: F-18 fluorodeoxyglucose uptake in primary cervical cancer as an indicator of prognosis after radiation therapy. Gynecol Oncol 101: 147-151, 2006.

43. Stefano A, Gallivanone F, Messa C, Gilardi MC and Castiglioni I: Metabolic impact of partial volume correction of $(18 \mathrm{~F})$ FDG PET-CT oncological studies on the assessment of tumor response to treatment. Q J Nucl Med Mol Imaging 58: 413-423, 2014.

44. Shankar LK, Hoffman JM, Bacharach S, Graham MM, Karp J, Lammertsma AA, et al: Consensus recommendations for the use of F-18-FDG PET as an indicator of therapeutic response in patients in national cancer institute trials. J Nucl Med 47: 10591066, 2006

45. Boellaard R, O'Doherty MJ, Weber WA, Mottaghy FM, Lonsdale MN, Stroobants SG, Oyen WJ, Kotzerke J, Hoekstra OS, Pruim J, et al: FDG PET and PET/CT: EANM procedure guidelines for tumour PET imaging: Version 1.0. Eur J Nucl Med Mol Imaging 37: 181-200, 2010

46. Grueneisen J, Schaarschmidt BM, Heubner M, Aktas B, Kinner S, Forsting M, Lauenstein T, Ruhlmann V and Umutlu L: Integrated PET/MRI for whole-body staging of patients with primary cervical cancer: preliminary results. Eur J Nucl Med Mol Imaging 42: 1814-1824, 2015.

47. Kurokawa T, Yoshida Y, Kawahara K, Tsuchida T, Okazawa H, Fujibayashi Y, Yonekura Y and Kotsuji F: Expression of GLUT-1 glucose transfer, cellular proliferation activity and grade of tumor correlate with (F-18)-fluorodeoxyglucose uptake by positron emission tomography in epithelial tumors of the ovary. Int J Cancer 109: 926-932, 2004.

48. Dhull VS, Sharma P, Sharma DN, Maharjan S, Suman Kc S, Patel C, Bal C and Kumar R: Prospective evaluation of 18F-fluorodeoxyglucose positron emission tomography-computed tomography for response evaluation in recurrent carcinoma cervix: Does metabolic response predicts survival? Int J Gynecol Cancer 24: 312-320, 2014. 